\title{
Minimal Standards for Electroencephalographic Laboratories
}

These guidelines for the minimal standards for Electroencephalographic Laboratories were developed by the Canadian Society of Clinical Neurophysiologists and passed at the Annual General Meeting in June 1990 in Banff.

\section{Preamble}

Until now, there have been no uniform, comprehensive guidelines for the operation of EEG laboratories across Canada. Some provinces have accreditation committees and written recommendations, while others have none. In those provinces without established minimal standards, the possibility of technically poor records and misleading interpretations is very real. This could lead to improper medical investigation and treatment and to unfortunate psychosocial consequences for the patient, e.g. with respect to employability or the legal operation of motor vehicles. Also, physical harm could occur to patients if technologists are not knowledgeable in electrical safety, the prevention of transmissible diseases, or in the use of volatile materials or sedative drugs which may be used in the performance of recordings. Furthermore, such laboratories should operate in a financially conscientious fashion, with respect to the health care systems of the provinces.

The minimal standards listed below are designed primarily for laboratories containing a single technologist in a town or city which is different from the one in which the electroencephalographer practices; i.e. peripheral EEG laboratories. The same standards should be met in larger, hospital-based laboratories. Separate guidelines may be necessary to address the more complex and/or invasive procedures implemented in large tertiary care centres and for some specialized services such as telephone transmission of EEGs and ambulatory EEG recordings.

The following standards were compiled by a subcommittee of the EEG section of the Canadian Society of Clinical Neurophysiologists (CSCN), which used established guidelines of the American Electroencephalographic Society, the British Columbia Medical Association, the Alberta Medical Association, the American Society of Electroneurodiagnostic Technologists, the Committee on Methods of Clinical Examination in Electroencephalography of the International Federation of Electroencephalography and Clinical Neurophysiology, the Neuroelectrophysiological Technologists of Ontario and the Canadian Association of Electroneurophysiology Technologists. The standards are subject to modification; the EEG Section of the CSCN welcomes suggestions.

\section{The Electroencephalographic Technologist}

A. Qualifications. A technologist working in a private, unsupervised EEG laboratory should have a registration certificate of the Canadian Board of Registration of Electroencephalographic Technologists. At least one year of further experience in an EEG laboratory containing one other registered EEG technologist and with an electroencephalographer in the same institution is recommended.

B. Continuing Education. The technologist should spend at least 5 days per year working in a supervised laboratory in a university educational/scientific meeting of the Canadian Association of Electroneurophysiology Technologists, the American Society of Electroneurodiagnostic Technologists or a provincial EEG technology meeting.

\section{The Electroencephalographer}

A. Qualifications. The electroencephalographer must be licensed to practice medicine in his/her province and must meet the qualifications for clinical electroencephalography stipulated by the Constitution of the CSCN (see Appendix).

B. Continuing Education. Electroencephalographers are encouraged to attend a refresher course in EEG or a scientific meeting of the Canadian Society of Clinical Neurophysiologists, the American EEG Society, the Canadian League Against Epilepsy or the American Epilepsy Society at least once every three years.

C. Other Aspects. The electroencephalographer should visit the laboratory a minimum of every six months.

\section{Technical Requirements}

A. Equipment. At least sixteen channel equipment is encouraged. Under no circumstances should less than eight channels of EEG data be recorded. The machine's leakage current and ground resistance should be checked annually and should meet the minimal standards of the Canadian Standards Association (CSA).

B. The full 21 electrodes recommended by the International Federation of Societies for Electro-encephalography and Clinical Neurophysiology should be used, except in prematures and neonates. The 10-20 system of electrode placement should be used, with head measurement to assure accurate placement. The electrode cap may be used, providing the calvarium is of normal shape and symmetry. A ground lead should be applied cephalad to the heart.

C. Disk electrodes should be used rather than needle electrodes. The use of needle electrodes is discouraged.

E. Inter-electrode impedances should be checked routinely before the recording commences and should be between 100 and 5,000 ohms. They should also be checked during the recording with the output shown on the tracing. Scarification of the skin should be avoided in order to reduce infection.

F. Using standard methods, the EEG electrodes should be cleaned or sterilized between patients.

G. Monitoring of the electrocardiogram and electro-oculogram is recommended. Monitoring of other biological parameters, such as electromyogram or respiration should be 
performed only when clinically indicated. The technologist who employs such techniques should be trained in their application and know the relevant clinical correlations.

H. A series of square wave calibrations should be used at the beginning and end of the recording, such that the largest signal produces a pen deflection of no less than $10 \mathrm{~mm}$ and the smallest signal produces a pen deflection of $2 \mathrm{~mm}$ or less. A minimum of 20 seconds of biocalibration should be used after the initial calibration. All channels should contain the same pair of widely spaced electrodes.

I. Both scalp-to-scalp (bipolar) and referential montages should be used. The electrode montage for each channel should be clearly indicated at the beginning of each montage as well as document any changes which occur during the test. Periods of eye opening and eye closure should be included in each montage, when feasible.

J. The standard recording should be obtained using filter settings which accurately reproduce signals from $0.5-70 \mathrm{~Hz}$. Paper speed of $30 \mathrm{~mm} / \mathrm{second}$ is standard. Sensitivity should usually be in the range of 5-10 microvolts/millimeter in adults. Filter settings and sensitivity should be recorded at the beginning of each montage and whenever they are changed during the recording. Less than 20 minutes of actual recording time is unacceptable.

K. Spontaneous sleep should be obtained whenever possible. At least one montage utilizing the midline, especially vertex, electrodes should be employed during sleep.

L. Intermittent photic stimulation should be performed as part of the routine procedure. Caution should be exercised if generalized or occipital epileptiform activity appears earlier in the recording and stimulation should be stopped immediately after a photoparaoxysmal response is obtained. Photic stimulation can be omitted in the elderly unless referring physician specifically requests it.

M. Overbreathing or hyperventilation should be used routinely unless contraindicated (e.g. severe cardiac or pulmonary disease, general infirmity, recent intracranial hemorrhage). Three minutes of overbreathing should be recorded as well as one and one-half minutes after cessation of hyperventilation on the same continued montage.

N. The technologist should obtain a history and a list of present medications from the patient and include these on a technologist sheet. The following information should also be documented on the front of the EEG record: Patient name, date of birth, patient identification number and date of recording.

\section{Telephone transmission of EEGs}

The above minimal standards apply. In addition: (i) the EEG technologist should receive specific training from others who are skilled and knowledgeable in this technology; (ii) a hard copy should be made; (iii) event markers or other signalling capability should be built in to the system to allow correlation of the transmitted with the original recording; (iv) there must be adequate documentation on the transmitted recording: patient identification, calibrations, changes in instrument controls and montages, patient behavior and activity; $(v)$ periodic checks of equipment should be performed to ensure adequacy of frequency response; and (vi) such recording should not be used for the determination of electrocerebral silence.

\section{Appendix}

Qualifications for clinical electroencephalography: the physician shall have F.R.C.P.C. or equivalent diploma in a medical specialty, or an F.R.C.S.C. or equivalent diploma in neurosurgery or a Ph.D. degree in a basic science area related to the field of testing performed. A minimum of six months of full time supervised training in EEG in a laboratory headed by a full member of (or an individual who qualifies for full membership for) the EEG Section of the CSCN or the American EEG Society is necessary to obtain such competence. 